\title{
Application Benefits of Advanced Equation-Based Multiphysics Modeling
}

\author{
Lars Langemyr and Nils Malm \\ COMSOL AB, Tegnérgatan 23, SE-111 40 Stockholm, Sweden \\ Contact author: Nils Malm, telephone +46 841295291 \\ nils@comsol.se
}

\begin{abstract}
In just the past few years, the field of mathematical modeling with equation-based tools has seen a number of advances and innovations that allow software developers to create programs that are at once far more powerful yet easier to use. To illustrate the benefits that users gain from the latest features and capabilities, this paper examines a problem where software that supports extended multiphysics calculates the applied voltage needed to produce a predefined thermally induced bending of a microscale device. The package used in this problem is FEMLAB, an equation-based environment for solving a large class of systems of coupled partial differential equations.

The software relies on a Galerkin discretization of a weak formulation of the PDEs using generic shape functions. Arbitrary couplings between equations are possible, and a correct discrete residual and Jacobian can be obtained for both linear and nonlinear problems. Therefore, the software can handle tightly coupled systems using an efficient all-at-once Newton solver or an implicit time-stepping method.

Linear systems are described by giving coefficient values for a generic PDE form. More general systems are specified as conservation laws where the user defines an arbitrary flux vector and source for each equation. In addition, the user has direct access to the underlying weak form, which adds additional flexibility to the system. For the less mathematically oriented user, predefined application templates set up the equations based on physical properties. The resulting equations can be viewed, modified, and arbitrarily coupled to other physics.

Direct access to the weak form allows fully integrated multidimensional modeling. Separate equations can be modeled on the boundary of a domain, or contributions can be added to the main equations. In particular, the weak form provides for the straightforward implementation of non-standard boundary conditions and those conditions connecting different types of physics, as is the case when doing, for example, fluid-structure interaction.

In traditional multiphysics problems, the interaction between fields is local in space. In addition to such local couplings, FEMLAB supports the use of non-local variables in any equation. We refer to that capability as extended multiphysics, and it can be used to model control systems, couple multidimensional models, couple discrete and semi-analytical models, solve inverse and optimization problems, and more. Non-local coupling variables can be defined through arbitrary coordinate mappings, as integrals over selected domains, or as integrals along a given direction. FEMLAB internally knows the sensitivity of the coupling variables with respect to the degrees of freedom, and therefore it can accurately compute the full Jacobian for extended multiphysics model.
\end{abstract}

